\title{
Study of Power Conversion Efficiency of a Novel Hybrid L-Band Erbium Doped Fiber Amplifier
}

\author{
Ricky Anthony \\ Heritage Institute of Technology \\ Chowbaga Road, Anandapur, \\ Kolkata, India
}

\author{
Rini Lahiri \\ National Institute of \\ Technology-Nagaland \\ Cachar, Assam, India
}

\author{
Sambhunath Biswas \\ Heritage Institute of Technology \\ Chowbaga Road, Anandapur, \\ Kolkata, India
}

\begin{abstract}
High gain and low noise figure are desired features of any erbium doped fiber amplifier (EDFA) based configuration. However, in $\mathrm{L}$ band region, low power and quantum conversion efficiencies have restricted EDFA based systems. The paper presents simulation studies of a novel EDFA based high L-band gain configuration which implements two different EDF's in bi-directionally pumped manner. The system shows a non-flattened high gain of $36.6 \mathrm{~dB}$ for an optimized net EDF of length $30 \mathrm{~m}$, pump power of $120 \mathrm{~mW}$ at $25^{\circ} \mathrm{C}$. It also presented a high power and quantum conversion efficiencies of $37.8 \%$ and $57.0 \%$, variations of which are also studied with input signal and pump powers.
\end{abstract}

\section{Keywords}

Erbium Doped Fiber Amplifier, Gain, Noise Figure, Power and Quantum Conversion Efficiency, L Band.

\section{INTRODUCTION}

Since the fabrication of erbium doped fiber in 1987 [1], a great deal of effort has been made to introduce newer high gain, low noise, high power conversion efficient and low cost configurations. L band optical communication using EDF based configurations along with dense wavelength division multiplexing (DWDM) has increased the capacity immensely. Today commercialized C and L band EDFA are available which can amplify 160 channels. However, low power and quantum conversion efficiencies (PCE and QCE) in L band have challenged researchers to report newer and better configurations. Compressions of backward output ASE power for gain tilt control, and gain enhancement are some of the methods [2, 3]. Others include inclusion of inline fiber grating laser for gain flatness and gain clamping. The gain spectrum of EDFA shows a non-linear, abrupt behavior in the $\mathrm{C}$ and $\mathrm{L}$ band $[4,5,6]$. The non-linear gain variation of an EDF has also encouraged gain flattening schemes such as MachZehnder filter [7], long period fiber grating [8] and side polished fiber based filter [9].

In this paper, a thorough investigation of PCE and QCE of an L-band hybrid configuration with different parameters has been reported.
As observed form figure 1, the system uses two different erbium doped fibers, EDF-II in between two EDF-I. The fiber amplifiers are pumped in a bidirectional manner by using $980 \mathrm{~nm}$ and $1480 \mathrm{~nm}$ laser pumps. An isolator implemented after the source, restrict the reflected power to affect the optical oscillator.

\section{EDFA MODELING}

According to Giles model [10], it was assumed that only homogeneous broadening occurs and ion-ion interaction is absent in an EDFA. It was further assumed that excited state absorption (ESA), pair induced quenching (PIQ) and Rayleigh backscattering (RBS) of the EDFA are negligible. The rate equations for forward (+) and backward (-) propagation for such a model is given by:

$$
\pm \frac{d P_{k}^{ \pm}}{d z}=\left(\alpha_{k}+g_{k}^{*}\right) \frac{n_{2} \mid a v}{\left.n_{t}\right|_{a v}} P_{k}^{ \pm}+g_{k}^{*} \frac{n_{2} \mid a v}{\left.n_{t}\right|_{a v}} m h f_{k} \Delta f_{k}\left(\alpha_{k}+l_{k}\right) P_{k}^{ \pm}
$$

The steady state population inversion equation will be given by:

$$
\frac{\left.n_{2}\right|_{a v}}{\left.n_{t}\right|_{a v}}=\frac{\sum_{k} \frac{P_{k}(z) \alpha_{k}}{h f_{k} \zeta}}{1+\sum_{k} \frac{P_{k}(z)\left(\alpha_{k}+g_{k}^{*}\right)}{h f_{k} \zeta}}
$$

Where, $\alpha_{\mathrm{k}}$ and $g_{k}^{*}$ are the absorption and gain coefficient of the erbium doped fiber. $\zeta$ and $1_{k}$ are the saturation parameter and background loss respectively. $P_{k}^{ \pm}$is the forward and backward propagating power of the $\mathrm{k}^{\text {th }}$ spectral component. Whereas $\left.n_{t}\right|_{a v}$ and $\left.n_{2}\right|_{a v}$ are the total average $\mathrm{Er}^{3+}$ ion concentration and that in the upper level. $h$ and $f_{k}$ are the Planck's constant and frequency of $\mathrm{k}^{\text {th }}$ spectral component. The configuration shown in figure 1 employs DWDM, separated by $50 \mathrm{GHz}$ channel spacing. The power/channel is varied for three different values to analyze its effects on power and quantum conversion efficiency. The isolators used have an insertion loss of $0.3 \mathrm{~dB}$, isolation of $20 \mathrm{~dB}$, input return loss of $60 \mathrm{~dB}$ and output return loss of $55 \mathrm{~dB}$. Two $980 \mathrm{~nm}$ pumps are used in a bidirectional manner for better noise figure advantage [11].

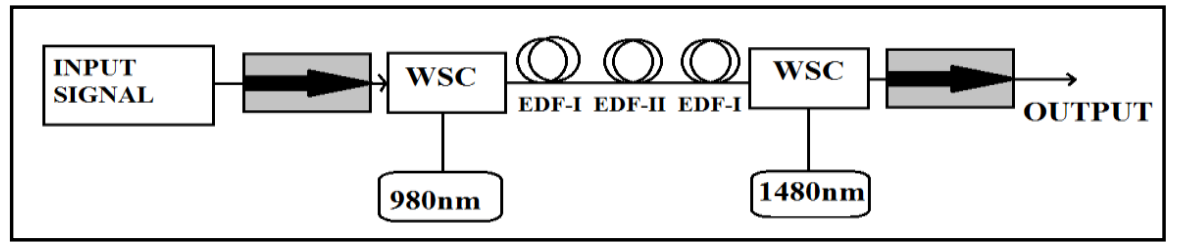

Fig 1: A novel L-band Hybrid EDFA configuration 
Bidirectional pumping provides an added advantage in terms of population inversion; small signal gain and provides prolonged uniformity compared to co-directional and counterdirectional pumping. The wavelength selective couplers (WSC) have signal and pump insertion losses of $0.2 \mathrm{~dB}$; pump and signal isolation of $20 \mathrm{~dB}$ and $30 \mathrm{~dB}$; signal and pump return losses of $50 \mathrm{~dB}$ and directivity of $55 \mathrm{~dB}$ respectively. Two different EDF's, EDF-I and EDF-II have saturation parameters $(\zeta)$ of $1.6050 \times 10^{16} / \mathrm{ms}$ and $3.091 \times 10^{15} / \mathrm{ms}$, with input and output splice losses of 0.3dB. EDF-I and EDF-II have absorption peaks of 3.5 and $23-27 \mu \mathrm{m}$ at $980 \mathrm{~nm}$. The absorption and emission spectrum of both the fibers are shown in figure 2 .

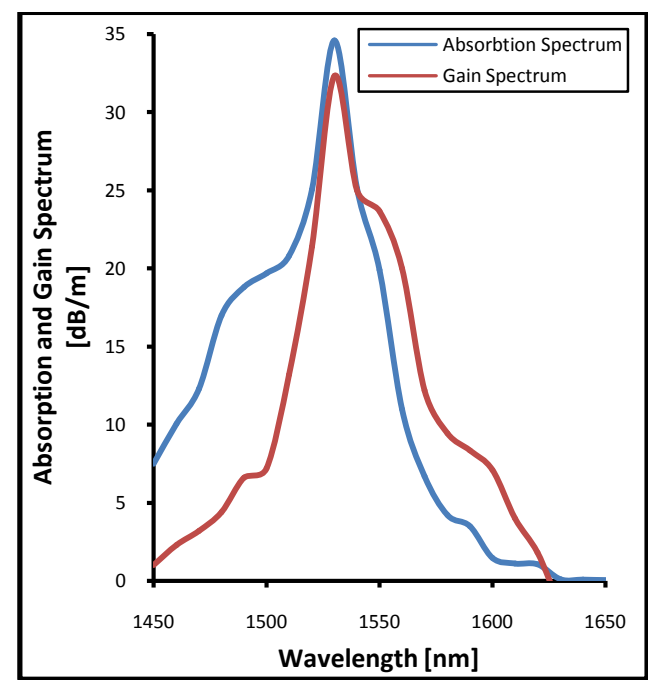

Fig 2: Absorption and Emission Spectra of EDF Type-I

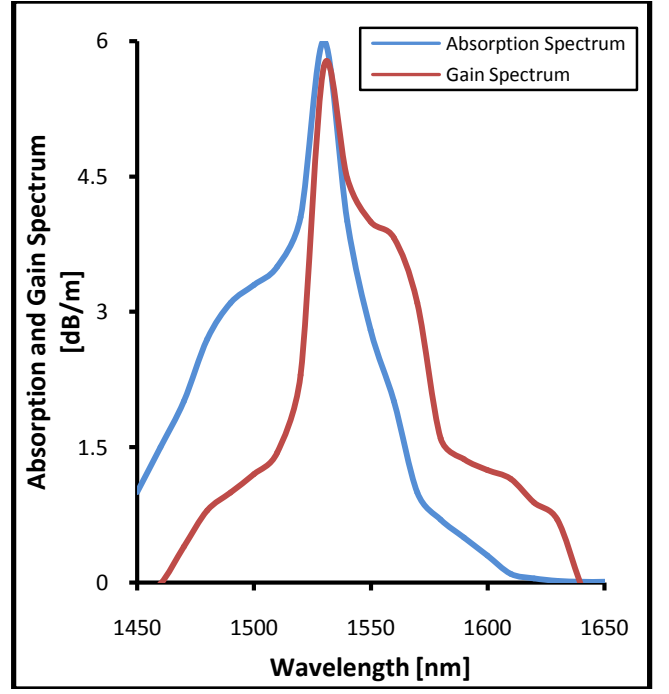

Fig 3: Absorption and Emission Spectra of EDF Type-II

\section{RESULTS AND DISCUSSIONS}

Booster amplifiers placed just after the input source needs to generate high output power to enhance the launched signal power. To obtain such a high output power, it is essential that large percentage of input light is converted into signal light. Power conversion efficiency is measure of pump power converted to signal power for amplification, a measure of system efficiency in terms of power, and is given by [12]:
Power Conversion Efficiency $(\mathrm{PCE})=\frac{\left(P_{\text {signal out }}-P_{\text {signal in }}\right)}{P_{\text {pump }}}$

The PCE reduces considerably in the $\mathrm{L}$ band region. Many newer and better configurations have been proposed, such as dual-band EDFA covering both $\mathrm{C}$ and L band [13]. The quantum conversion efficiency (QCE) on the other hand, is more direct measure of efficiency in terms of photon transfer from pump to signal is given by:

Quantum Conversion Efficiency $(\mathrm{QCE})=$ PCE $x \frac{\lambda_{\text {signal }}}{\lambda_{\text {signal }}}$

Also, the non-uniformity of gain is another factor which limits the number of channel a system can transmit. A gain over $20 \mathrm{~dB}$ and noise figure below $5 \mathrm{~dB}$ is considered ideal for optical communication. Such a gain increases optical signal to noise ratio (OSNR) and reduces bit-error-rate (BER), which is appropriate for receiver. Hence, for systems feasibility, these are important parameters to be analyzed.

\subsection{Gain, PCE and QCE versus wavelength for Different pump powers}

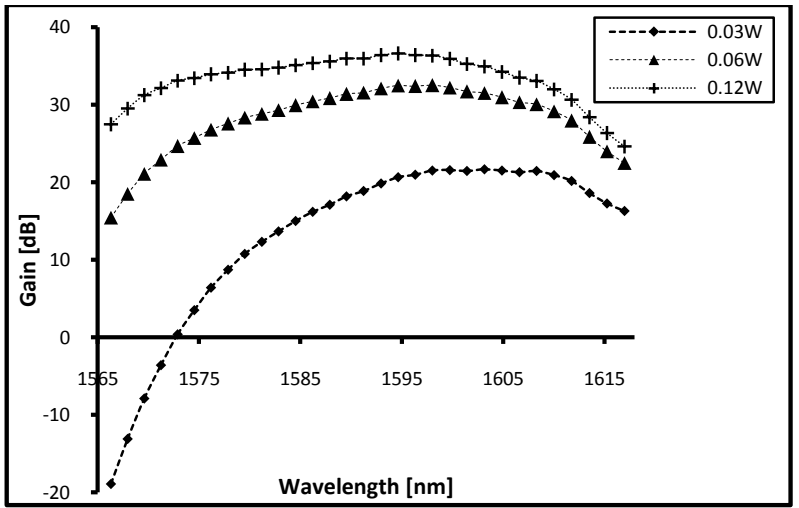

Fig 4: Dependence of Gain with Wavelength for different pump powers of pumps-I and II

Simulation studies of gain variation for pumps-I and II with three different pump powers for both the $980 \mathrm{~nm}$ pumps: $30 \mathrm{~mW}, 60 \mathrm{~mW}$ and $120 \mathrm{~mW}$ (As shown in figure 4) have been discussed. At $30 \mathrm{~mW}$ pump powers, average gain obtained was at $18.23 \mathrm{~dB}$, with a maximum at $21.68 \mathrm{~dB}$ a poor gain tilt of $29.4 \mathrm{~dB}$. The power and quantum conversion efficiency was as low as $3.38 \%$ and $5.54 \%$. On doubling the pump power to $60 \mathrm{~mW}$, PCE and QCE increased by 7 times to $23.03 \%$ and $37.63 \%$. The maximum gain too showed a sharp rise, to $32.52 \mathrm{~dB}$ with a gain tilt and net average gain of $6.3 \mathrm{~dB}$ and $29.5 \mathrm{~dB}$ respectively. When compared to $60 \mathrm{~mW}$, at $120 \mathrm{~mW}$, PCE and QCE increased approximately 1.5 times to reach $33.28 \%$ and $54.26 \%$, respectively and an average gain of $34.10 \mathrm{~dB}$ with a gain tilt of $-1.771 \mathrm{~dB}$ was obtained. The figure 5 shows the variation of PCE and QCE for different values of pump powers. The PCE and QCE show an exponential rise up to $60 \mathrm{~mW}$, which then attains saturation for higher pump powers. PCE and QCE reach values as high as $32.45 \%$ and $52.95 \%$. At such a condition, EDFA can operate as power amplifiers without signal distortion and cross-talk. 


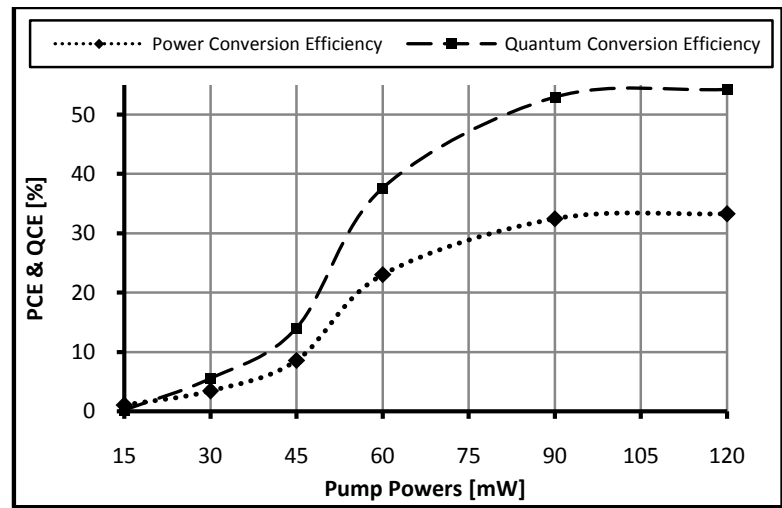

Fig 5: Variation of Power and Quantum Conversion Efficiency variation with pump powers

\subsection{Gain and N.F versus Wavelength for Different Input Signal Power}

Figure 6, shows the gain curve with varying signal power/ channel for different signal wavelengths. At lower ends of L band region, there is a steep fall of EDFA gain, but at higher wavelength region the gain fall is gradual. With increase in signal power, inversion is depleted, this reduces the gain considerably and increases noise figure (N.F), as shown in figure 7. As the signal output values exceed saturation values noise figure rises sharply. At higher input power signal, depletion of inversion occurs which can no longer be compensated by the pump. The signal robs gain from the backward amplified spontaneous emission (ASE), reducing gain and increasing N.F. And at even higher input signal power level, the noise figure attains a near constant value.

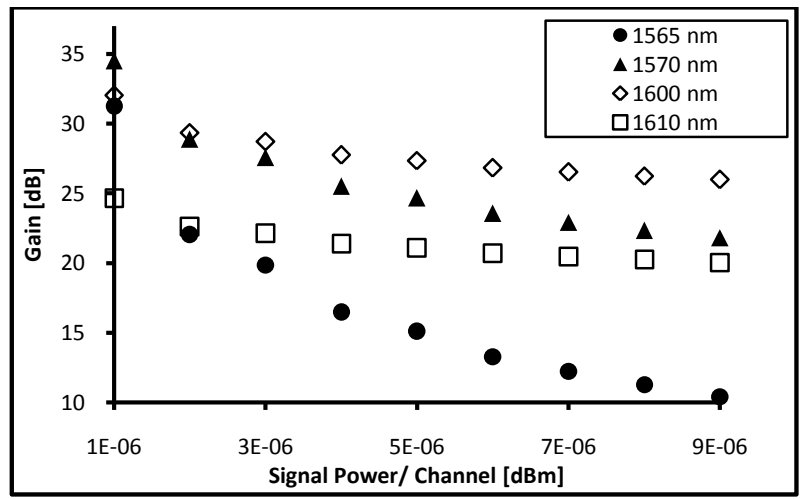

Fig 6: Gain variation with Signal power/Channel for different signal wavelengths

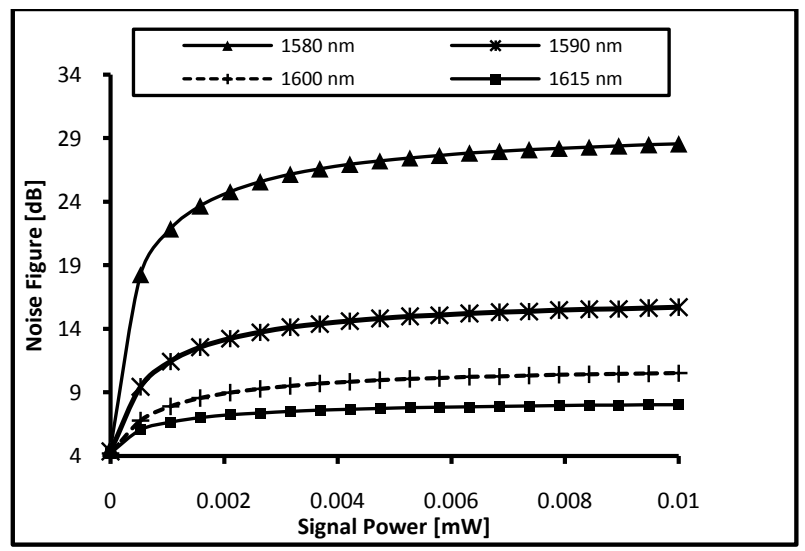

Fig 7: Noise Figure Variation with input signal powers for different signal wavelengths

\subsection{Power and Quantum Conversion Efficiency for variable Amplifier Lengths}

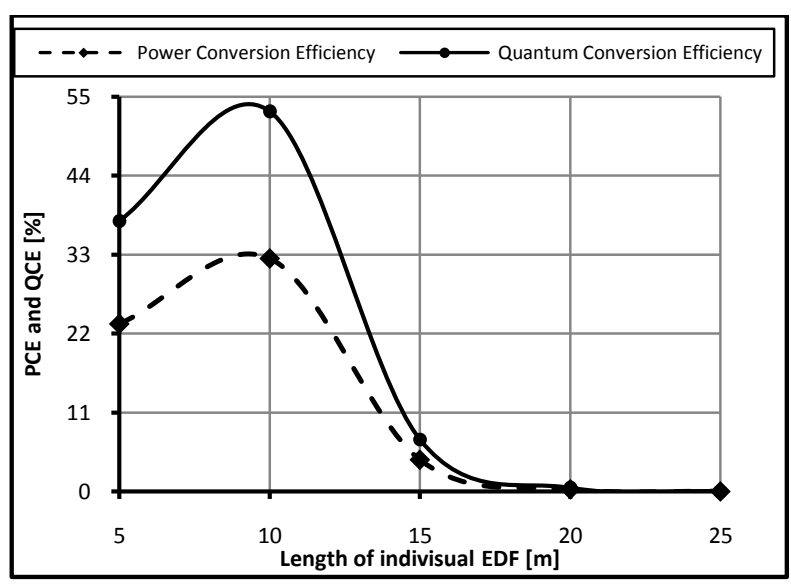

Fig 8: Variation of Power and Quantum Conversion Efficiency with amplifier length

As shown in figure 8 , both power and quantum conversion efficiencies show a sharp increase with increase in length of individual erbium doped fibers (EDF's). For individual EDF lengths of $5 \mathrm{~m}(15 \mathrm{~m}$ in total), PCE and QCE values are $23.40 \%$ and $37.68 \%$, which increased to a maximum of $32.70 \%$ and $52.95 \%$ for $10 \mathrm{~m}$ EDF lengths, respectively. Such an increase in PCE and QCE is extremely encouraging, since it provides better inversion ratio and output gain. However, as the EDF length is increased further, PCE and QCE falls off monotonically to $4.43 \%$ and $7.27 \%$ at $15 \mathrm{~m}$ individual EDF lengths and continues to decrease, eventually reaching near zero values at $20 \mathrm{~m}$ EDF length. As evident from the figure, at $10 \mathrm{~m}$, the fiber reaches maximum inversion and any further increase above the optimum EDF length with constant pump power introduces ASE noise, without any considerable increase in gain. High power conversion efficiency in $65-80 \%$ has been reported in $\mathrm{C}$ band region [14]. Considerable increase in Aluminum doping and optimizing numerical apertures accordingly can result in further improvement as [15]. However increasing $\mathrm{Er}^{3+}$ ion concentration results in quenching effects and hence, reduces power and quantum conversion efficiencies.

\subsection{Power and Quantum Conversion} Efficiency for variable Input Signal Powers

With constant EDF lengths and pump powers of $10 \mathrm{~m}$ and $120 \mathrm{~mW}$ respectively, the power and quantum conversion efficiencies with input signal powers has been plotted in figure 9. It may be observed from the figure that as signal power increases, initially more and more pump power is transformed to amplify the signal wavelength, hence both PCE and QCE at -40dBm increase gradually from $9.36 \%$ and $1.18 \%$ respectively to a steady value of $36.63 \%$ and $56.93 \%$ at $-20 \mathrm{dBm}$. With further increase in the signal power, saturation occurs and inversion is reduced, resulting in a constant PCE and QCE, as output. However, above Odbm, the PCE and QCE start to fall; this is so because the erbium ions now undergo absorption instead of emission. Hence there is a point beyond which any increase in input signal power will not be efficient in terms of power and gain. 


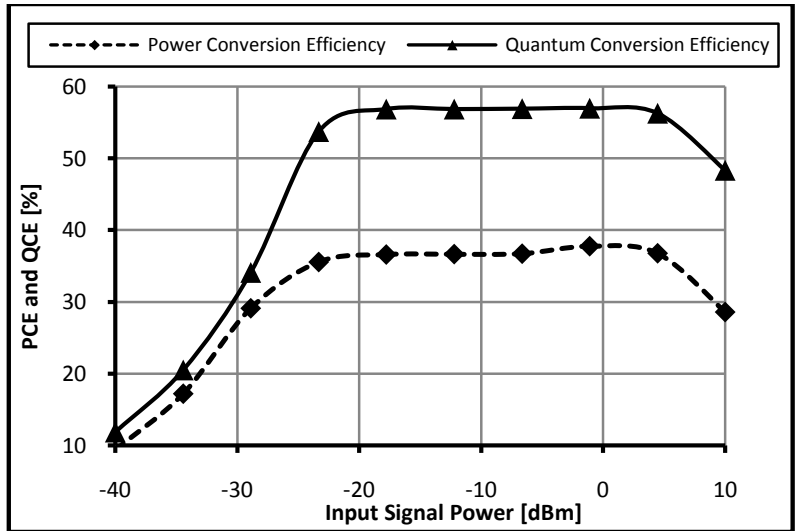

Fig 9: Power and Quantum Conversion Efficiencies with varying input signal power

\section{CONCLUSIONS}

An attempt has been made in this paper to investigate the power and quantum conversion efficiencies of a novel $\mathrm{L}$ band EDFA, which implements two EDF's having different saturation, absorption and emission spectra. As evident from the figures, the configuration provides a high gain of $34.01 \mathrm{~dB}$ at $120 \mathrm{~mW}$ pump powers. The system also depicts an improved power and quantum conversion efficiencies, reaching as high as $32.70 \%$ and $52.95 \%$ from $10 \mathrm{~m}$ amplifier length. The paper also discusses the saturation effects of the system in terms of PCE and QCE with input signal power, where it remained constant at $36.63 \%$ and $56.93 \%$. It has always been a challenge to increase the PCE at L band optical communication with EDFA. Further improvement in PCE and QCE is possible with rightful use of Aluminum (Al) concentration and host composition.

Table 1. Power Conversion and Quantum Conversion Efficiencies with input signal power

\begin{tabular}{|c|c|c|}
\hline $\begin{array}{c}\text { Input Signal } \\
\text { Power (dBm) }\end{array}$ & PCE (\%) & QCE (\%) \\
\hline-40.0 & 9.36 & 11.8 \\
\hline-34.45 & 17.23 & 20.5 \\
\hline-28.89 & 2.91 & 34.1 \\
\hline-12.23 & 36.6 & 56.94 \\
\hline-1.11 & 37.8 & 57.0 \\
\hline 10 & 28.6 & 48.3 \\
\hline
\end{tabular}

Table 2. Power Conversion and Quantum Conversion Efficiencies with pump power

\begin{tabular}{|c|c|c|}
\hline $\begin{array}{c}\text { Pump Power } \\
(\mathbf{m W})\end{array}$ & PCE (\%) & QCE (\%) \\
\hline 15 & 0.99 & 0.164 \\
\hline 30 & 3.382 & 5.548 \\
\hline 45 & 8.556 & 14.011 \\
\hline 60 & 23.031 & 37.632 \\
\hline 90 & 32.456 & 52.948 \\
\hline 120 & 33.285 & 54.26 \\
\hline
\end{tabular}

\section{ACKNOWLEDGMENTS}

The authors would like to thank Prof. Prabir Banerjee and Prof. Siladitya Sen of Department of Electronics and Communication Engineering, Heritage Institute of Technology and Prof.S.Baishya of National institute of Technology-Silchar for their encouragement and suggestions during the progress of the work.

\section{REFERENCES}

[1] R.J. Mears, L. Reekie, I.M. Jaundice, and D.N. Payne, "High gain rare earth doped fiber amplifier at $1.54 \mu \mathrm{m}$. Optical Fiber Communication Conference, vol.3, 1987.

[2] Bumki Min, Hosung Yoon and Won Jae Lee," Coupled structure for wide-band EDFA with gain and noise figure improvements from C to L-band ASE injection". IEEE Photon. Technol. Lett., vol.12, no.5, pp.480-482, May 2000.

[3] Yanbin Zhang, et al, "Wavelength and power dependence of injected C-band laser on pump conversion efficiency of L-band EDFA". IEEE Photon. Technol. Lett, vol.14, pp.290-292, March 2002.

[4] S. Yamashita and M. Nishihara, "L-band erbium doped fiber amplifier incorporating an inline fiber grating laser". IEEE Journal of Selected Topics Quantum Electron., vol.7, pp. 44-48, Jan-Feb. 2001.

[5] M.A. Mahdi, P. Poopalan, et al., "All optical gain locking in erbium doped fiber amplifiers using doublepass super fluorescence". IEEE Photon. Technol. Lett., vol.11, no. 12, pp. 1581-1583, Feb 1999.

[6] R. Anthony, S.N. Biswas, "Gain flattening and noise figure analysis of a dual stage bowtie WDM EDFA Configuration in C-band". Intern. J. Scien. \& Engg. Research (IJSER), vol.3, Iss.4, 2012.

[7] J.Y. Pan, M.A. Ali, A.F. Elrefaie and R.E. Wagener,' Multi-wavelength fiber amplifier cascades with equalization employing Mach-Zehnder optical filter", IEEE Photon. Technol. Lett., vol.7, p.p1501-1503, 1995.

[8] A.M. Vengsarkar, P.J. Lemaire, J.B. Judkins, V. Bhatia, T. Erdogan and J.E. Sipe, "Long period fiber gratings as band rejection filters". IEEE J. Lightwave Technol., Vol.14, pp.58-65, 1996.

[9] R.K. Varshney, B. Nagaraju, A. Singh, B.P. Pal and A.K.Kar, "Design and realization of all fiber broadband tunable gain equalization filter for DWDM Signals". Optical Express, vol.15, pp. 13519-13530, 2007.

[10] C.R. Giles and E. Desurvire, "Modeling erbium-doped fiber amplifiers". IEEE J. Lightwave Technol., vol.9, pp. 271-283, 1992.

[11] R.G. Smart et. al., "Experimental comparison of 980nm and $1480 \mathrm{~nm}$ pump saturated in-line erbium doped fiber amplifier suitable for long-haul soliton transmission systems". Photonics Technology Letters, vol.5 iss.7, pp.770-773, 1993.

[12] B. Pedersen, M.L. Dakss and W.J. Miniscalco, "Conversion efficiency and noise in erbium-doped fiber power amplifiers". Optical Fiber Amplifier and Their Applications, vol.13, OSA Technical Digest Series, pp.170-176, 1991.

[13] S. Hwang et. al., "Comparative high power conversion efficiency of C-plus L-band EDFA". IEEE Elec. Lett., vol.37, no.25, pp.1539-1541, 2001.

[14] J.F. Massicott, R. Wyatt, B.J. Ainslie and S.P. CraigRyan, "Efficient, high power, high gain, $\mathrm{Er}^{3+}$ doped silica fibre amplifier". Electron. Lett., vol.26, no.14, 1990.

[15] Jian Liu, Paul.F. Wysocki, Matthew Andrejco and Bera Palsdottir". SPIE Optical Engineering, vol.40, no.7, 2001. 